\section{ORIGINAL RESEARCH}

\section{J.M. Wood}

B. Kundu

A. Utter

T.A. Gallagher

J. Voss

V.A. Nair

J.S. Kuo

A.S. Field

C.H. Moritz

M.E. Meyerand

V. Prabhakaran

\title{
Impact of Brain Tumor Location on Morbidity and Mortality: A Retrospective Functional MR Imaging Study
}

BACKGROUND AND PURPOSE: fMRI is increasingly used in neurosurgery to preoperatively identify areas of eloquent cortex. Our study evaluated the efficacy of clinical fMRI by analyzing the relationship between the distance from the tumor border to the area of functional activation (LAD) and patient preand postoperative morbidity and mortality.

MATERIALS AND METHODS: The study included patients with diagnosis of primary or metastatic brain tumor who underwent preoperative fMRI-based motor mapping $(n=74)$ and/or language mapping $(n=77)$. The impact of $L A D$ and other variables collected from patient records was analyzed with respect to functional deficits in terms of morbidity (paresis and aphasia) and mortality.

RESULTS: Significant relationships were found between motor and language $L A D$ and the existence of either pre- or postoperative motor $(P<.001)$ and language deficits $(P=.009)$. Increasing age was associated with motor and language deficits $(P=.02$ and $P=.04$ respectively). Right-handedness was related to language deficits $(P=.05)$. Survival analysis revealed that pre- and postoperative deficits, grade, tumor location, and LAD predicted mortality. Motor deficits increased linearly as the distance from the tumor to the primary sensorimotor cortex decreased. Language deficits increased exponentially as the distance from the tumor to the language areas decreased below $1 \mathrm{~cm}$. Postoperative mortality analysis showed an interaction effect between motor or language LAD and mortality predictors (grade and tumor location, respectively).

CoNCLUSIONS: These findings indicate that tumors may affect language and motor function differently depending on tumor LAD. Overall, the data support the use of $\mathrm{FMRI}$ as a tool to evaluate patient prognosis and are directly applicable to neurosurgical planning.

\footnotetext{
ABBREVIATIONS: BOLD = blood oxygen level-dependent; $\mathrm{Cl}=$ confidence Interval; $\mathrm{EPI}=$ echoplanar imaging; $\mathrm{fMRI}=$ functional $\mathrm{MR}$ imaging; $\mathrm{HR}=$ hazard ratio; $\mathrm{LAD}=$ lesion to activation distance; $\mathrm{OR}=$ odds ratio; $\mathrm{SE}=$ standard error; $\mathrm{SMC}=$ sensorimotor cortex; SSDI = Social Security Death Index; WHO = World Health Organization
}

$\mathbf{0}$ ngoing refinements in fMRI technology, functional paradigms, and postprocessing methods have led to increased reliability and use of fMRI as a noninvasive clinical tool for preoperative localization of eloquent cortex in relation to pathology. Validation in multiple studies has led to increased use of fMRI for routine preoperative assessment of patients with brain lesions. ${ }^{1}$ There has been consistent agreement between fMRI and the more invasive Wada testing for language lateralization in numerous studies. ${ }^{2,3}$ fMRI offers the benefits of

Received October 7, 2010; accepted after revision December 7.

From the Department of Neuroradiology, University of Wisconsin School of Medicine and Public Health, University of Wisconsin-Madison, Madison, Wisconsin.

J.M. Wood and B. Kundu contributed equally to this work.

This work was support by NIH grant UL1RR025011.

Paper previously presented at: Annual Meeting of the American Society of Neuroradiology and the Neuroradiology Education and Research Foundation, May 15-24, 2010; Boston, Massachusetts; and Annual Meeting of the American Society of Functional Neuroradiology; February 24-26, 2010; Las Vegas, Nevada

Please address correspondence to: Joel M. Wood, MD, University of Wisconsin School of Medicine and Public Health, 750 Highland Ave, Madison, WI 53705; e-mail: jwood2@ wisc.edu; or Bornali Kundu, Health Emotions Research Institute, 6001 Research Park Blvd., Madison, WI 53719; e-mail: bkundu@wisc.edu

Indicates open access to non-subscribers at www.ajnr.org

Indicates article with supplemental on-line tables at www.ajnr.org

Indicates article with supplemental on-line photos at www.ajnr.org http://dx.doi.org/10.3174/ajnr.A2679 spatial localization of sensorimotor and language centers, which can pre-emptively impact decision-making. As a result, there has been continued interest in the role of fMRI not only for localization purposes but also as a predictor of postoperative patient deficits based on the proximity of fMRI activation to the margin of tumor or other lesions.

Currently, patients harboring lesions encroaching on functional centers will typically undergo awake craniotomy with electrical stimulation mapping. Haglund et $\mathrm{al}^{4}$ showed, in a mapping study of 40 patients with dominant hemisphere temporal lobe gliomas, that a distance of $>1$ $\mathrm{cm}$ from the nearest language center to the resection margin resulted in significantly fewer permanent postoperative language deficits. Not only did Haglund et al confirm the conclusions of Ojemann et $\mathrm{al}^{5}$ and others concerning the wide variation in location of cortical language centers in the brain and the unreliability of anatomic landmarks, ${ }^{6}$ they also provided insight into balancing aggressive resections while minimizing postoperative deficits. Use of fMRI as a noninvasive preoperative tool to predict similar outcomes has clear decision-making benefits. Yetkin et $\mathrm{al}^{7}$ examined fMRI in the preoperative setting and suggested a relationship between increased tumor encroachment on the SMC and an increase in pre- and postoperative motor deficits. In this study, we explored the role of fMRI in predicting postsurgical morbidity as well as mortality related to tumor lesions encroaching on both language areas and the SMC. 


\begin{tabular}{|c|c|c|c|c|}
\hline Characteristics of Patients & $<1 \mathrm{~cm}$ & $1-2 \mathrm{~cm}$ & $>2 \mathrm{~cm}$ & $P$ Value \\
\hline \multicolumn{5}{|c|}{ Based on distance between lesion and SMC } \\
\hline No. of subjects ( $n=74)$ & 31 & 16 & 27 & .09 \\
\hline Male (\%) & 55 & 50 & 74 & .20 \\
\hline Handedness ( $\%$ right $)^{a}$ & 74 & 93 & 76 & .34 \\
\hline Mean age $\pm S D(y r)$ & $50 \pm 17.0$ & $43 \pm 12.5$ & $41 \pm 15.5$ & .05 \\
\hline Grade III or IV tumors (\%) & 71 & 50 & 43 & .10 \\
\hline Mean length of follow-up $(m o)^{b}$ & 40 & 35 & 49 & .28 \\
\hline \multicolumn{5}{|c|}{ Based on distance of lesion from Broca or Wernicke area ${ }^{\mathrm{C}}$} \\
\hline No. of subjects $(n=77)$ & 26 & 15 & 36 & .01 \\
\hline Male $(\%)$ & 61 & 40 & 55 & .74 \\
\hline Handedness (\% right) & 81 & 73 & 78 & .88 \\
\hline Mean age \pm SD (years) & $44 \pm 14.4$ & $40 \pm 18.6$ & $49 \pm 15.7$ & .12 \\
\hline Grade III or IV tumors (\%) & 54 & 60 & 53 & .89 \\
\hline Mean length of follow-up $(\mathrm{mo})^{b}$ & 58 & 56 & 43 & .26 \\
\hline
\end{tabular}

a For the motor deficits study cohort, handedness was unknown for $n=5$ subjects.

${ }^{b}$ The survival analysis patient cohort is slightly different from the cohort used in other parameter characterizations.

${ }^{\mathrm{c}}$ Grouping was based on minimum distance from either Broca or Wernicke area.

\section{Materials and Methods}

\section{Study Subjects}

Subjects were selected from a database of 423 patients who underwent fMRI as part of presurgical planning at the University of Wisconsin, Madison between June 1999 and July 2009. Inclusion criteria for this study selected all patients with a diagnosis of primary or metastatic tumors in any lobe of the brain and who also underwent motor and/or language mapping using fMRI. Table 1 and On-line Tables 1 and 2 include demographic information. Patients gave informed consent according to the study protocol approved by the local review board. Patient information was collected from medical records. Any record of preoperative or postoperative weakness (lower extremity, upper extremity, and/or facial) and/or aphasia (Broca and Wernicke areas, conduction, global, and so forth) was included in the analysis. This means that only gross motor deficits and aphasia were considered. No other specific neuropsychological testing was done.

Separate analysis was performed with preoperative and postoperative deficits combined and also just considering postoperative deficits (On-line Tables 2 and 3). Mortality information was collected for all patients by using medical records that were crossreferenced with the SSDI. Original data from Social Security Administration accessed via the http://stevemorse.org/ssdi/ssdi.html Web site, aggregates information from the following Web sites: ancestry.com, familysearch.com, familytreelegends.com, geneaology.com, geneaologybank.com, newenglandancestors.org, rootsweb.com, and worldvitalrecords.com. ${ }^{8}$ Patients with no listing in SSDI were considered still alive.

\section{Paradigms}

The language and motor paradigms used to assess patients are described in more detail in Moritz and Haughton. ${ }^{9}$ In brief, activation of the Broca area is best seen with word-generation fMRI tasks. This study used 2 types of word-generation tasks: 1) alternating 20-second blocks of antonym word generation versus rest, and 2) alternating 20 -second blocks of letter-word-generation versus rest. Wernicke area was identified with alternating 20 -second blocks of text-readingversus-symbols task. In this task, the patient silently read a short paragraph in the text-reading block. During the control or symbols block, the patient was shown a paragraph of symbols and asked to scan and find specific symbols. The symbols block controlled for eye movements during reading, which presumably helped discriminate visual and eye movement-related activity from the true language areas. Motor activation was determined using a range of tasks including unilateral finger tapping, alternating hand finger tapping, unilateral foot/ankle movement, and/or tongue/lip movement versus rest. Four alternating 20-second blocks were used for only one task. Multiple tasks were used because not all patients showed activation with any one task. We selected the task that resulted in robust or the largest amount of activation for each subject because not all patients performed all tasks.

\section{fMRI Acquisition and Processing}

Imaging was done with either a $1.5 \mathrm{~T}$ or $3 \mathrm{~T}$ commercial MR imaging scanner (Sigma GE Healthcare, Milwaukee Wisconsin) equipped with high-speed gradients. BOLD-weighted single-shot EPIs were obtained repeatedly at intervals defined by the TR for each patient during task performance. Technical parameters were the following: FOV, $24 \mathrm{~cm}$; matrix, $64 \times 64$; TR, $2000 \mathrm{~ms}$; TE, $40 \mathrm{~ms}$ (for $1.5 \mathrm{~T}$ ) or $27 \mathrm{~ms}$ (for $3 \mathrm{~T}$ ); flip angle, $85^{\circ}$ (for $1.5 \mathrm{~T}$ ) or $75^{\circ}$ (for $3 \mathrm{~T}$ ); 6-mm coronal plane sections (for $1.5 \mathrm{~T}$ ) or $5-\mathrm{mm}$ axial plane sections (for $3 \mathrm{~T}$ ). Spatial coverage was sufficient to provide mapping of the entire cortex. The number of images and the length of imaging varied with the paradigm. Imaging duration ranged from 3 to 5 minutes. Additional highresolution anatomic scans, including $3 \mathrm{D}$ volumetric T1- and T2weighted sequences were acquired as part of the preoperative assessment. See On-line Supplementary Section 1 for detailed postprocessing methods. All postprocessing was performed with the AFNI software (http://afni.nimh.nih.gov/). ${ }^{10}$ Activation was determined by cross-correlation of the time-course of the EPI signal intensity at each voxel with a generalized least-squares-fitting algorithm to a smoothed and temporally delayed boxcar reference function modeling the presumed hemodynamic response. This comparison provided a voxelwise $t$ statistic, with which images were thresholded individually to optimize visualization of language or motor areas and were overlaid on the coregistered anatomic brain volume maps.

Thresholding was subjectively applied with the intent of optimizing specificity and sensitivity to expected regions of task response. This meant adjusting the threshold to minimize spurious voxels that were considered artifacts (eg, due to head motion). It also involved adjusting the threshold to highlight the expected responses (eg, Broca area or primary SMC) at a level that displayed a typical suprathreshold extent. This was subjectively adjusted to localize a particular gyrus 


\begin{tabular}{|c|c|c|c|}
\hline Characteristic & No Deficits & Deficits & $P$ Value \\
\hline \multicolumn{4}{|l|}{ Motor } \\
\hline Sex (\% male) & 57 & 65 & .63 \\
\hline Age (mean) & 41 & 50 & .02 \\
\hline Handedness (\% right) & 77 & 76 & .99 \\
\hline High-grade $^{a}$ & 49 & 65 & .24 \\
\hline \multicolumn{4}{|l|}{ Language } \\
\hline Sex (\% male) & 60 & 60 & .86 \\
\hline Age (mean) & 43 & 50 & .04 \\
\hline Handedness (\% right) & 69 & 90 & .05 \\
\hline High-grade $^{a}$ & 44 & 69 & .06 \\
\hline
\end{tabular}

a High-grade tumors include grades III and IV on the WHO scale.

or region, which represented a statistical probability of greatest confidence as indicated by the $t$ statistical overlay. Often, a compromise threshold was applied to balance the need to highlight an expected response with the concern of minimizing artifacts. For example, in a dataset that exhibited significant task-correlated head motion, it may not have been possible to minimize the artifacts while still retaining sufficient sensitivity to the presumed task-related responses. The taskrelated response magnitudes were also dependent on factors such as the patient's ability to perform a particular fMRI task or whether the BOLD response was compromised by the presence of tumor. Thus, the threshold was subjectively varied for each individual fMRI scan on the basis of the quality of the data and medical mapping concerns.

\section{Image Analysis and Interpretation:}

Images used in the analysis were compiled at the time of surgery by a trained MR imaging technologist (C.M.) and were used by the neurosurgical team for presurgical planning. T1-weighted, T2-weighted, and contrast-enhanced T1-weighted structural images were analyzed in PACS. Broca area included Brodmann areas 44, 45, 47, and 12. Wernicke area included Brodmann area 22. The shortest distance in any plane (coronal, sagittal, or transverse) from the periphery of the tumor to the border of the area of functional activation was measured using PACS (On-line Figure 1). Tumor edge was defined as the enhancing margin for tumors that enhance with contrast on T1weighted images or the peripheral margin of the solid portion of the tumor as noted in T2-weighted images (versus the edematous areas). For low-grade tumors where the margin appeared more heterogeneous on T2 fluid-attenuated inversion recovery, we used the T1weighted images. These distances were then categorized according to an a priori decision that was based on data from past literature, ${ }^{3,6}$ which found these cutoff values to be of clinical relevance. The categories were the following: 1) $<1 \mathrm{~cm}, 2$ ) between 1 and $2 \mathrm{~cm}$, and 3) $>2$ $\mathrm{cm}$. To assess the influence of a large number of predictor variables, we initially performed univariate analysis on all variables of interest and then subsequent multivariate analysis with potential confounding factors (covariates) and factors of interest. $\chi^{2}$ tests were used to compare categoric variables.

Logistic and Cox proportional hazards models were used to ex- plore relationships between deficits and survival, respectively. The baseline for the survival analysis was the date of clinical fMRI, and we computed the number of days survived from fMRI to July 2010. Predictors with $P<.1$ (1-sided) in univariate analysis were included in multivariate models. Model diagnostics such as goodness-of-fit tests and residual analyses were obtained to assess whether model assumptions were satisfied, and corrective action such as data transformation was pursued if warranted. $P<.05$ (2-sided) was the criterion for statistical significance. All analyses were done by using R statistical software $^{11}$ or the Statistical Package for the Social Sciences (SPSS, Chicago, Illinois).

\section{Results}

Of the 423 patients who underwent $\mathrm{AMRI}$ for presurgical planning and satisfied the inclusion criteria of primary or metastatic tumors, 77 patients had tumors encroaching the language area and had fMRI data for language tasks, and 74 patients with tumors encroaching on the motor area had fMRI data for motor tasks. Median follow-up in the clinic was 1337 days (range, 78-3367 days). There were more patients in the $>2 \mathrm{~cm}$ LAD category for the language subset of patients $(P=$ $.01)$. There were no differences in sex, handedness, or tumor grade in LAD categories across motor and language subsets $(P>.05)$. There were older patients in the $<1 \mathrm{~cm}$ LAD category for the motor subset $(P=.05)$. Older patients were also in the increased-deficits category for both the motor and language subset populations ( $P=.02, P=.04$, respectively). Grade was trending toward significance in association with language deficits $(P=.06$, Tables 1 and 2$)$.

\section{Univariate Analysis}

Significant associations were found between SMC LAD and the existence of paresis $(P<.001)$ and between language LAD and the existence of aphasia $(P=.009$; Table 3$)$. Moreover, there was a linear relationship between LAD and the existence of motor deficits. Specifically, the incidence of weakness decreased by approximately $36 \%$ from LAD of $<1 \mathrm{~cm}$ to LAD between 1 and $2 \mathrm{~cm}$, and then decreased again by $39 \%$ for LAD of $>2 \mathrm{~cm}$ (Fig 1). This differed from the relationship observed between tumor location and aphasia. Here, the incidence of aphasia was much higher for distances of $<1 \mathrm{~cm}$ and then did not vary beyond this cutoff (Fig 1). To confirm that the trends seen in the language data were not artifacts of combining the deficits from both Broca and Wernicke areas, we separately examined the relationship between LAD and the incidence of aphasia with respect to Broca and Wernicke areas and found the trend to be consistent for the Broca area $(P=.01)$ but not significant for the Wernicke area $(P=.28$, On-line Table 4$)$.

\section{Multivariate Analysis}

Multiple logistic regression showed LAD to be the best predictor of postoperative motor deficits in a model including both

Table 3: Univariate analysis: relationship between $L A D$ and the existence of language and motor deficits

\begin{tabular}{lccc}
\hline Characteristic & $<1$ & $1-2$ & $2+$ \\
\hline $\begin{array}{l}\text { SMC LAD vs motor deficits } \\
\quad \text { With pre- or postoperative paresis (\%) }\end{array}$ & $84(\mathrm{Cl}, 66-94)$ & $50(\mathrm{Cl}, 28-72)$ & $11(\mathrm{Cl}, 1-28)$ \\
$\begin{array}{l}\text { Broca and/or Wernicke area LAD vs language deficits } \\
\text { With pre- or postoperative aphasia (\%) }\end{array}$ & $68(\mathrm{Cl}, 48-83)$ & $33(\mathrm{Cl}, 15-58)$ & $28(\mathrm{Cl}, 16-44)$ \\
\hline
\end{tabular}




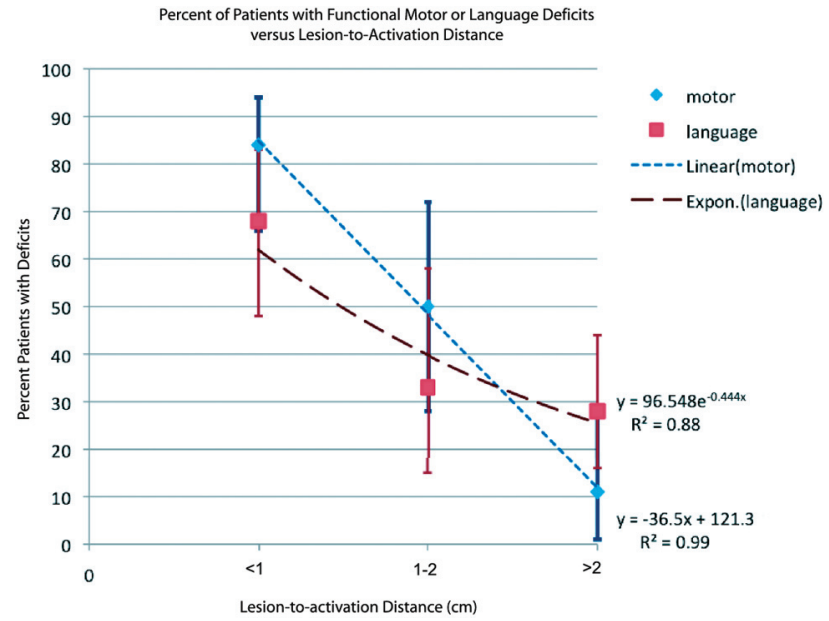

Fig 1. Dissociation between motor and language deficits according to distance between tumor and eloquent cortex. The data suggest a linear relationship $\left(R^{2}=0.99\right)$ between distance from the tumor to the area of activation and the existence of motor deficits (red) and an asymptotic relationship (exponential fit, $R^{2}=0.88$ ) between the distance from the tumor to the area of activation and the existence of language deficits (blue). Error bars depict 95\% Cls calculated for a proportion.

LAD and age. Only LAD of $>2 \mathrm{~cm}$ was statistically significant $(\beta=3.2 ; \mathrm{SE}=1.12$; change in $\mathrm{OR}=26.6$; $\mathrm{CI}, 3-233 ; P=$ $.003)$. LAD of $1-2 \mathrm{~cm}(\beta=0.14$; $\mathrm{SE}=0.69$; change in $\mathrm{OR}=$ 1.15; CI, $0.29-4.47 ; P=.84)$ and LAD of $<1 \mathrm{~cm}(\beta=1.14$; $\mathrm{SE}=1.1 ; P=.83$ this level was used as reference) were not significant. However, the effect may be clinically relevant seen through the effect directions and CIs. Age moderately predicted deficits $(\beta=-0.04 ; \mathrm{SE}=0.02$; change in $\mathrm{OR}=0.96$; CI, $0.92-1 ; P=.08)$. OR of weakness for a patient of average age ( 44 years) was 1.01 for lesion distances between 1 and $2 \mathrm{~cm}$ (relative to distances of $<1 \mathrm{~cm}$ ) and 0.05 for distances of $>2$ $\mathrm{cm}$ (relative to distance of $>1 \mathrm{~cm}$ ). Change in OR of deficit versus no deficit with increase in age (per year) was 1.04.

Similar analysis of the language deficit subset showed LAD to be the most significant predictor of aphasia (inclusive of all types: Broca, Wernicke, and nonspecific aphasia) in a model including LAD, grade, and age. Grade and age were moderate predictors of the incidence of aphasia. The OR for deficit in a patient of average age (44 years) and high-grade tumor was 0.60 for lesion distances between 1 and $2 \mathrm{~cm}(\beta=-1.42$; SE, 0.86 ; change in $\mathrm{OR}=0.24$; CI, $0.04-1.30 ; P=.10)$ and 0.48 for distances of $>2 \mathrm{~cm}(\beta=-1.65 ; \mathrm{SE}=0.67$; change in $\mathrm{OR}=$ 0.19 ; CI, $0.05-0.71 ; P=.01)$ relative to lesion distances of $<1$ $\mathrm{cm}(\beta=-0.57 ; \mathrm{SE}=1.04 ; P=.58)$. Change in OR for deficit versus no deficit for a low-grade tumor was $0.26(\beta=-1.33$; $\mathrm{SE}=0.61 ; \mathrm{CI}, 0.08-0.87 ; P=.02$; reference level $\mathrm{LAD}=<1$ $\mathrm{cm})$ and for age (per increase of 1 year) was $1.03(\beta=0.03$; $\mathrm{SE}=0.02$; CI, 0.99-1.07; $P=.09$; reference level LAD $=<1$ $\mathrm{cm})$.

We also examined the influence of LAD on preoperative and postoperative deficits separately. The results for preoperative motor deficits mirrored those of postoperative motor deficits $(P<.001$ for both). The results for preoperative language deficits, however, did not $(P=.12$ for preoperative, $P=$ .01 for postoperative) mirror postoperative language deficits.

\section{Survival Analysis}

On-line Figure 2 shows the Kaplan-Meier curves for the motor data, indicating that survival time for patients with either preoperative or postoperative motor deficits was significantly longer (mean $=2233$ days, CI, 1958-2508 days) than for patients with both preoperative and postoperative motor deficits (mean = 1544 days; CI, 1046-2042; Mantel-Cox logrank test, $\left.\chi^{2}=5.61, P=.018\right)$. Cox proportional hazards model, used to analyze the influence of multiple factors (On-line Table 5), indicated both preoperative and persistent postoperative motor deficits $(\mathrm{HR}=2.624$; CI, 0.987-6.978; $\mathrm{HR}=3.159$; CI, $1.183-8.435$, respectively) to be predictive of mortality. Grade was highly predictive of mortality $(P<.001)$ for the motor deficit patient population. We also found a significant effect of tumor lobe in the motor deficit population $(\mathrm{HR}=3.172$; $\mathrm{CI}$, 1.081-9.310 when comparing mortality of patients with parietal lobe tumor versus those with frontal lobe tumor).

Similar analyses were performed for the language-patient population. Kaplan-Meier analysis indicated that survival time for patients with either preoperative or postoperative deficit was longer (mean $=2306$ days; CI, 2013-2599 days) than that for patients with both preoperative and postoperative deficits ( mean $=1282$ days; CI, 847-1718 days); however, this difference only trended toward significance (Mantel-Cox logrank test, $\chi^{2}=3.08, P=.079$; On-line Figure 3 ). The Cox proportional hazards model was trending toward significance with respect to language deficits $(\mathrm{HR}=1.993$; $\mathrm{CI}, 0.909-$ $4.370)$. Grade was highly predictive of mortality $(P<.001)$ for the language-patient population. Postoperative mortality was not significantly related to motor or language LAD or tumor hemispheric location. We explored a number of models, including interaction between these parameters (On-line Table 5 ). While some of the models significantly described variance in the data, the effect sizes were weak between individual factors. There was a significant interaction effect between language LAD and grade $(P<.001)$; however, this may have been mainly driven by the strong effects of grade as indicated by the results of univariate analysis relating grade and mortality. Motor LAD and lobe interaction were trending toward significance $(P=.06)$, but the effect of the individual factors was not strong. Other interactions tested included LAD by either motor or language deficits; however, none of these models significantly captured the data.

\section{Discussion}

fMRI methods offer insight into relationships between lesions and their functional consequences. Imaging is a key preoperative tool for neurosurgeons and its use is correlated with improved surgical outcomes, such as decreasing incidence of functional deficits, when used as part of surgical planning. ${ }^{1,12-16}$ A predictor of functional deficits is the proximity of the lesion to eloquent cortex; indeed, past imaging studies have shown that distances of $>1 \mathrm{~cm}$ between tumor edge and the functional cortex correlate with significantly fewer motor and language deficits. ${ }^{4,7}$ The validity of these imaging studies has been confirmed by intraoperative cortical stimulation mapping. ${ }^{1,4,17}$ Bizzi et al $^{18}$ showed that fMRI had high overall sensitivity $(83 \%)$ and specificity $(82 \%)$ for detecting functional cortex adjacent to a focal mass lesion, with languagearea detection being slightly less sensitive and specific com- 
pared with the motor area. They also found lower sensitivity for high- (III and IV) compared with low-grade gliomas. However, very few studies address this distance relationship in the context of functional imaging and compare motor with language deficits. Furthermore, there are no studies, to our knowledge, that searched for an association between lesion location and patient mortality. This information would allow surgeons to inform patients about possible outcomes before performing any procedure.

This study focused on patients with primary and metastatic brain tumors who had undergone functional imaging to isolate individual language and motor areas. The relationship between LAD and the incidence of functional deficits such as weakness and aphasia as well as survival was assessed. We looked at a possible association between tumor proximity and a number of other factors such as age and sex. There was a significant association between deficits and the distance from the tumor margin to the SMC and to language areas, but language and motor deficits showed different trends. We found that prevalence of motor deficits increased linearly approximately $39 \%$ from LAD of $>2 \mathrm{~cm}$ to LAD between 1 and $2 \mathrm{~cm}$ and approximately $34 \%$ more as LAD continued to decrease below $1 \mathrm{~cm}$. This is consistent with findings published by Yetkin et al (1998). ${ }^{7}$ However, there was a distinctly nonlinear trend found in the language data. Here, prevalence of aphasia decreased dramatically from LADs of $<1 \mathrm{~cm}$ to LADs between 1 and $2 \mathrm{~cm}$ and then leveled off for all LADs of $>2 \mathrm{~cm}$. These results may be due to underlying differences in the organization of SMC-versus-language areas.

There is high individual variability in language representation compared with motor representation within the eloquent cortex. ${ }^{4,6,18,19}$ Electrical-mapping studies have questioned traditional views regarding distinct language areas such as Broca and Wernicke areas and suggest that language areas are smaller and more discrete in individuals and that damage to language-related tracts, such as the arcuate fasciculus, may significantly influence language deficits. ${ }^{4,6,20}$ This finding may explain the trend we found in the current study, in which close proximity to major language areas is highly correlated with existence of aphasia, but as proximity decreases, the overall language network is minimally disrupted. Thus, there is a stable probability of aphasia not related to LAD. Most interesting, the trend is only significant for the distance from Broca area LAD, but not Wernicke area LAD and is perhaps due to differences in the anatomic distribution of these two functionally separate areas. Broca area is thought to be more distinct compared with the Wernicke area, and perhaps the relationship between encroachment on the Wernicke area and aphasia is more complex than can be captured with this analysis. Our results confirm that the distance between tumor and eloquent cortex is the best predictor of postoperative outcomes in patients and that age and tumor grade are also significant predictors. Thus, patients can be evaluated to some degree by these factors before intracortical mapping, to give an idea of what to expect for their individual case.

Patient mortality was not significantly associated with proximity of tumor to language or motor areas on its own; however, interactions between language $\mathrm{LAD}$ and grade were significant and interactions between motor LAD and lobe were trending toward significance. In the case of the patient group with motor deficits, parietal lobe tumors predicted mortality. This effect may be consistent with studies of patients with parietal lobe lesions, with neglect showing poor recovery. ${ }^{21,22}$ As expected, mortality was predicted by grade in both patients with language and motor deficits. Further validation of these results is needed with a prospective study, larger sample sizes, and different patient cohorts.

A possible limitation of the study was that the threshold was set on an individual basis rather than at a group level. This was done because the same tasks evoke variable amounts of activation in individuals. All images were assessed by the same technologist; this practice added consistency to data acquisition and processing. The clinicians used these thresholded maps for presurgical planning, which we hypothesized would influence patient morbidity and mortality. Therefore, we used individually thresholded maps in our analysis to examine factors influencing patient morbidity and mortality. Motion artifacts and tumor-induced vascular uncoupling may distort the BOLD signal intensity; however, we used software to correct for these factors. A subset of patients also underwent breath-holding scanning, which provided confidence in the degree of neurovascular coupling adjacent to the tumor present in these patients.

Another limitation of this study is our minimal consideration of white matter tracts. Further analysis would include other types of imaging, such as diffusion tensor imaging, to analyze the influence of lesions on these tracts. Finally, our sample size was not determined by an a priori sample-size calculation; instead, we used all the available subjects satisfying the inclusion criteria. Despite our being able to find significant differences, it is possible that our study is underpowered to detect some clinically significant differences.

Also, we attempted to examine the relationship between preoperative and postoperative deficits with respect to tumor proximity (LAD) separately. The results for preoperative motor deficits mirrored those found with postoperative deficits; this finding suggests that surgery did not significantly alter morbidity in these patients. The results for preoperative language deficits, however, $\operatorname{did} \operatorname{not}(P=.12$ for preoperative, $P=$ .01 for postoperative) mirror postoperative language deficits. The results suggest that surgery altered morbidity in these patients as predicted by LAD. There is also the influence of other language areas such as the supplementary motor area, where lesions at or near the area may also influence language deficits. ${ }^{17}$

Overall, this study gives clinicians and surgeons a comparison of the prevalence of motor-versus-language deficits in relation to lesion proximity from eloquent cortex for patients with primary or metastatic brain tumors. It also provides some evidence regarding mortality with regard to tumor location and other lesion-related parameters. Additionally, it supports the literature, providing a basis for the use of tumor proximity as a valid predictor of postoperative outcomes.

\section{Conclusions}

This study provides further evidence supporting a relationship between tumor proximity to eloquent cortex as determined by fMRI and the existence of functional deficits. There was a difference between this relationship for motor and language areas. There was a graded association between the distance from 
tumor to the primary SMC and the incidence of weakness, while there was an asymptotic relationship between the distance from tumor to language areas and the incidence of aphasia. In the latter case, there is a threshold of $1 \mathrm{~cm}$ beyond which distance does not affect the incidence of aphasia. Distance between tumor and eloquent cortex may be used as an initial prognostic indicator on a patient-by-patient basis.

\section{Acknowledgments}

We thank Alejandro Munoz del Rio, MD, for his advice regarding the statistical analyses in this study.

Disclosures: Veena Nair, Research Support (including provision of equipment or materials). Grants, Details: NIH RC Grant; Wallace Coulter Foundation; Institute for Clinical and Translational Research, University of Wisconsin-Madison.

\section{References}

1. Haberg A, Kvistad KA, Unsgard G, et al. Preoperative blood oxygen leveldependent functional magnetic resonance imaging in patients with primary brain tumors: clinical application and outcome. Neurosugery 2004;54:902-15

2. Yetkin FZ, Swanson S, Fischer M, et al. Functional MR of frontal lobe activation: comparison with Wada language results. AJNR Am J Neuroradiol 1998;19:1095-98

3. Sabbah P, Chassoux F, Leveque C, et al. Functional MR imaging in assessment of language dominance in epileptic patients. Neuroimage 2003;18:460-67

4. Haglund MM, Berger MS, Shamseldin M, et al. Cortical localization of temporal lobe language sites in patients with gliomas. Neurosugery 1994;34:567-76

5. Ojemann G, Ojemann J, Lettich E, et al. Cortical language localization in left, dominant hemisphere. J Neurosurg 1989;71:316-26

6. Sanai N, Mirzadeh Z, Berger MS. Functional outcome after language mapping for glioma resection. $N$ Engl J Med 2008;358:18-27

7. Yetkin FZ, Ulmer JL, Mueller W, et al. Functional magnetic resonance imaging assessment of the risk of postoperative hemiparesis after excision of cerebral tumors. International J Neuroradiol 1998;4:253-57

8. Quinn J, Kramer N, McDermott D. Validation of the Social Security Death
Index (SSDI): an important readily available outcome database for researchers. West J Emerg Med 2008;9:6-7

9. Moritz $\mathrm{CH}$, Haughton V. Functional MR imaging: paradigms for clinical preoperative mapping. Magn Reson Imaging Clin N Am 2003;11:529-42

10. Cox R. AFNI: software for analysis and visualization of functional magnetic resonance neuroimages. Comput Biomed Res 1996;29:162-73

11. R Core Development Team. R: A Language and Environment for Statistical Computing. 2.10.1 ed. Vienna, Austria; R Foundation for Statistical Computing; 2008

12. Petrella JR, Shah LM, Harris KM, et al. Preoperative functional MR imaging localization of language and motor areas: effect on therapeutic decision making in patients with potentially resectable brain tumors. Radiology 2006;240: 793-802. Epub 2006 Jul 20

13. Wu JS, Mao YM, Zhou LF, et al. Clinical evaluation and follow-up outcome of diffusion tensor imaging-based functional neuronavigation: a prospective, controlled study in patients with gliomas involving pyramidal tracts. Neurosurgery 2007;61:935-49

14. Khan RB, Gutin PH, Rai SN, et al. Use of diffusion weighted MRI in predicting early post-operative outcome of a new neurological deficit after brain tumor resection. Neurosurgery 2006;58:60-66, discussion 60-66

15. Sunaert S. Presugical planning for tumor resectioning. J Magn Reson Imaging 2006;23:887-905

16. Tieleman A, Deblaere K, Roost DV, et al. Preoperative fMRI in tumour surgery. Eur Radiol 2009;19:2523-34. Epub 2009 May 9

17. Nelson L, Samir L, Haughton VM, et al. Preoperative mapping of the supplementary motor area in patients harboring tumors in the medial frontal lobe. J Neurosurg 2002;97:1108-14

18. Bizzi A, Blasi V, Falini A, et al. Presurgical functional MR imaging of language and motor functions: validation with intraoperative electrocortical mapping. Radiology 2008;248:579-89. Epub 2008 Jun 6

19. Ojemann GA. Brain organization for language from the perspective of electrical stimulation mapping. Behav Brain Sci 1983;6:189-230

20. Warren JE, Crinion JT, Lambon Ralph MA, et al. Anterior temporal lobe connectivity correlates with functional outcome after aphasic stroke. Brain 2009; $132: 3428-42$

21. Gialanella B, Ferlucci C. Functional outcome after stroke in patients with aphasia and neglect: assessment by the motor and cognitive functional independence measure instrument. Cerebrovasc Dis 2010;30:440-47

22. Verhoeven CL, Post MW, Schiemanck SK, et al. Is cognitive functioning 1 year poststoke related to quality of life domain. J Stroke Cerebrovasc Dis 2010 Aug 31. [Epub ahead of print] 